Historic, archived document

Do not assume content reflects current scientific knowledge, policies, or practices. 



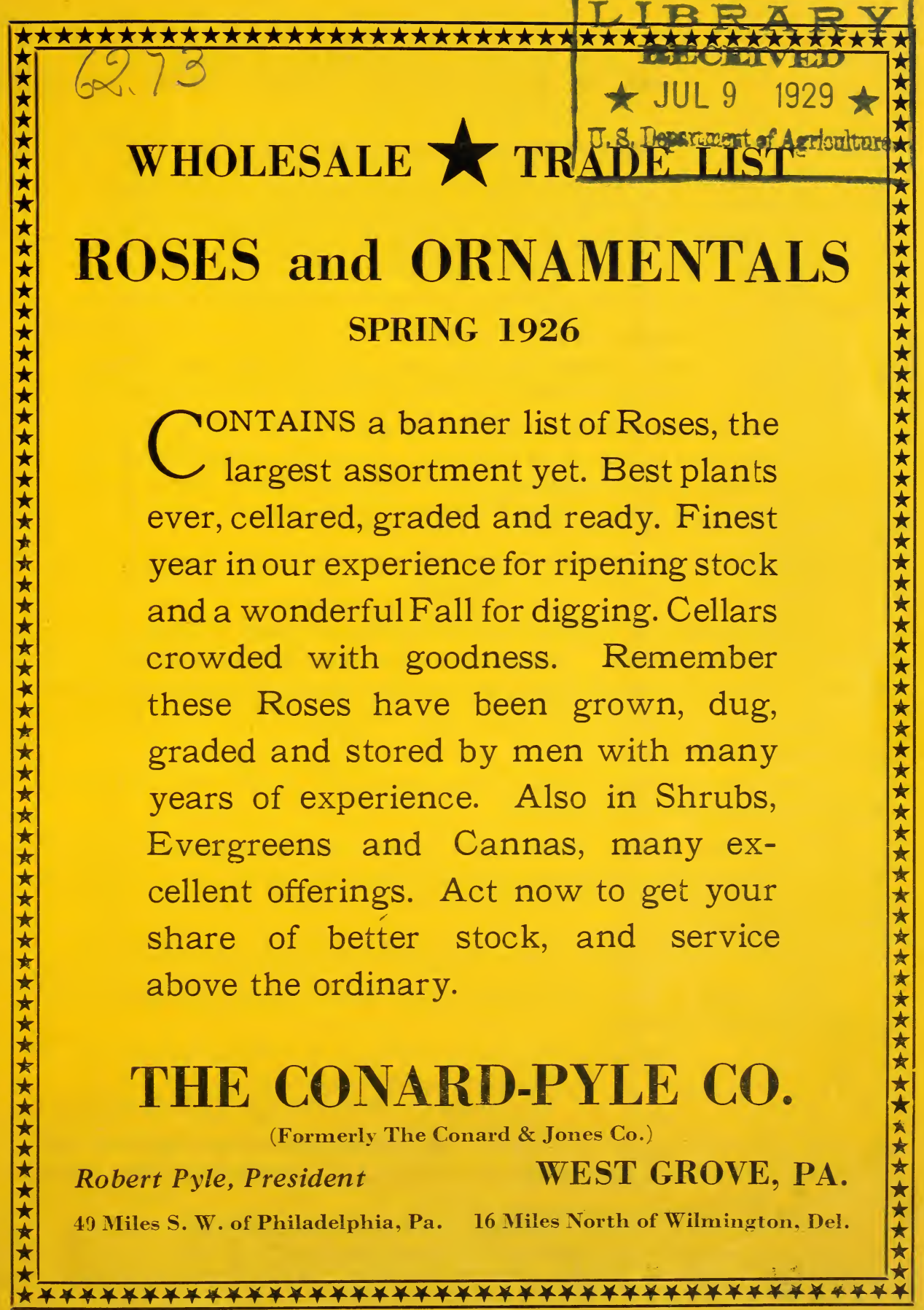




\section{WHOLESALE TRADE LIST SPRING 1926}

\section{GETTING THE MOST FOR YOUR MONEY}

The material we offer in this List is, mainly, first hand. You get extra value, then, because the stock is fresh, clean and plump with energy and vitality aplenty. You get extra value because the goods can be shipped direct to you from our nursery, not having to lose any of their desirability through the careless handling of middlemen. You get extra value because your order is cared for in an interested, efficient manner by skilled men. Therefore, it should pay you to buy from this List, because the extra value you get will mean extra profits for you. Why not check up on your wants and send us your order! You'll want more!

\section{TERMS}

All prices net f. o. b. West Grove; packing at cost. 250 or more at 1000 rate; 25 at 100 rate. Orders accepted on condition they shall be void should injury befall stock from frost or other causes beyond our control.

Shipments travel at purchaser's risk. Claims, if any, must be made on receipt of goods.

Terms cash; except to those with established credit, who may make special arrangements for payment.

We endeavor to keep varieties true to name and will replace, on proper proof, any not true; but we do not guarantee the success of the grower. In event of untrue stock our liability must be limited to invoice value at time of shipment.

Copy of Pennsylvania State Certificate of Inspection on all orders.

When writing, use your business letter-head or state definitely your connection with the trade.

Members American Association of Nurserymen.

Eastern Nurserymen's Association.

Pennsylvania Nurserymen's Association, etc.

All quotations, prices, contracts and grading based on Horticultural Standards adopted by the American Association of Nurserymen, June, 1923.

\section{THE CONARD-PYLE CO., WEST GROVE, PA.}

(The Conard \& Jones Co. became The Conard-Pyle Co. January 1st, 1925)

Robert Pyle, Pres. R. T. Satterthwait, Vice-Pres. E. P. Groff, Sec'y-Treas. 


\section{WHOLESALE PRICE LIST SPRING 1926}

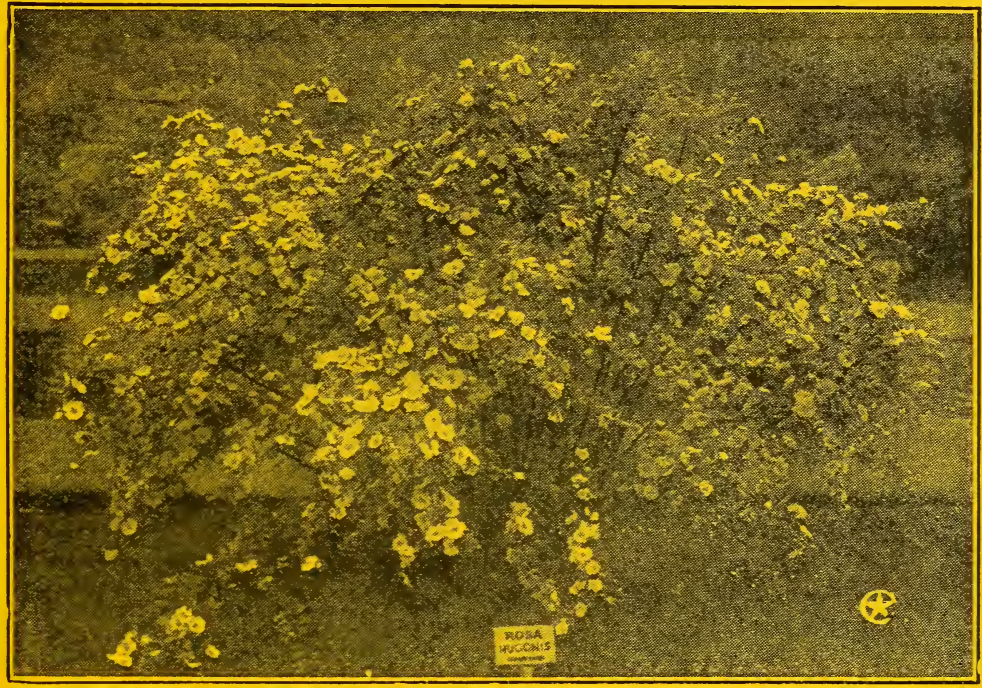

"Best hardy yellow Rose we know of, and most popular species in America"-Dr. E. H. Wilson, Arnold Arboretum

\section{Rosa Hugonis Worth Getting Next! "Golden Rose of China"}

In actual use for more than ten years, Hugonis has proved all we claim for it-completely hardy, exceptionally early bloomer, beautiful flowers and foliage, wide range of usefulness. No matter how much you have liked to handle other Roses, you will be mighty well-pleased with the practical money-making results Hugonis will bring you.

You run no risk. The leaders among American Nurserymen have handled Hugonis regularly, from the first, getting their supply here. This last Fall brought repeat orders by the score. Hugonis must be goodit must be SELLING BIG. The plants now for sale are freshly dug, ownroot, field grown and mighty nice stuff.

In the last ten years the supply has sold out early, in spite of increased production. Better get yours ordered today. Will be pleased to take care of you!

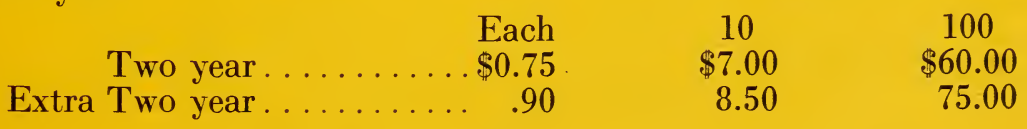




\section{THE CONARD-PYLE CO. WEST GROVE, PA. ROBERT PYLE, President}

\section{ROSES - HARDY CLIMBERS}

Strong 2 year No. 1 Plants, except as noted

Own-root and budded. Will be shipped with tops trimmed, unless otherwise specified. If desired uncut, remit $\$ 2$ extra per hundred.

\begin{tabular}{|c|c|c|c|c|}
\hline & 1 & 10 & 100 & 1000 \\
\hline American Pillar, pink. & 0.50 & $\$ 4.00$ & $\$ 30.00$ & $\$ 250.00$ \\
\hline 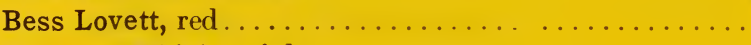 & .50 & 4.00 & 35.00 & \\
\hline 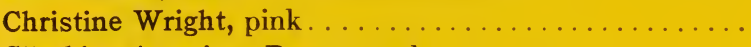 & .50 & 4.00 & 35.00 & \\
\hline Climbing American Beauty, red $\ldots \ldots \ldots \ldots \ldots \ldots$ & .50 & 4.00 & 35.00 & 300.00 \\
\hline Climbing American Beauty, red No. $2 \ldots \ldots \ldots \ldots \ldots$ & .30 & 2.50 & 20.00 & \\
\hline 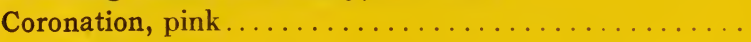 & .50 & 3.50 & 25.00 & \\
\hline Dr. Huey, red..... & .75 & 6.00 & 50.00 & \\
\hline 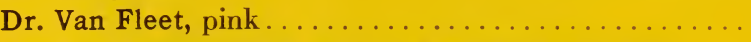 & .50 & 4.00 & 30.00 & 250.00 \\
\hline Dorothy Perkins, pink, strong, 1 yr. field, $21 / 2-3 \mathrm{ft} \ldots \ldots$ & .25 & 2.00 & 15.00 & \\
\hline 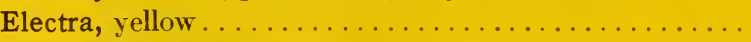 & .40 & 3.00 & 25.00 & \\
\hline Emily Gray, yellow . . . . . . . . . . . & .75 & 6.00 & 50.00 & \\
\hline herry red No.2............. & .25 & 2.00 & 18.00 & \\
\hline 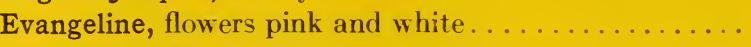 & .40 & 3.00 & 25.00 & \\
\hline ( & .50 & 4.00 & 30.00 & 250.00 \\
\hline Hiawatha, brilliant crimson. . ................ & .30 & 2.50 & 20.00 & \\
\hline 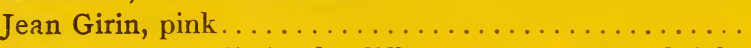 & .35 & 2.50 & 18.00 & \\
\hline 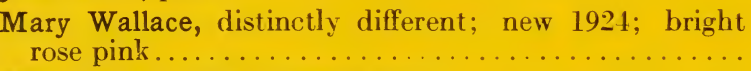 & .75 & 7.00 & 60.00 & \\
\hline May Queen, clear bright pink................ & .40 & 3.00 & 25.00 & \\
\hline Walsh, white..... & .50 & 3.50 & 25.00 & \\
\hline . & .50 & 3.50 & 25.00 & \\
\hline Paul's Scarlet Climber, red. & .50 & 4.00 & 35.00 & 300.00 \\
\hline et Climber, red No. $2 \ldots \ldots \ldots \ldots \ldots \ldots$. & .30 & 2.50 & 20.00 & \\
\hline Philadelphia, red . . . . . . . . . . . . . . . . & .50 & 3.50 & 25.00 & \\
\hline Prof. C. S. Sargent, yellow . ................... & .40 & 3.00 & 22.00 & 200.00 \\
\hline Roserie, red Tausendschon No. $2 \ldots \ldots \ldots \ldots \ldots$ & .25 & 2.00 & 18.00 & \\
\hline $\begin{array}{l}\text { Tausendschon, white and pink No. } 2 . \quad \text { (Also No. } 3 \\
\quad @ 12 \text { per } 100) \ldots \ldots \ldots \ldots \ldots \ldots \ldots \ldots \ldots \ldots \ldots \ldots \ldots \ldots \ldots\end{array}$ & .25 & 2.00 & 18.00 & \\
\hline Veilchenblau, reddish lilac.................. & .50 & 3.50 & 25.00 & \\
\hline Wichuraiana, white $\ldots \ldots \ldots \ldots \ldots \ldots \ldots \ldots \ldots$ & .50 & 3.50 & 25.00 & \\
\hline
\end{tabular}

\section{EVERBLOOMING CLIMBERS}

2 year No. 1 budded Plants

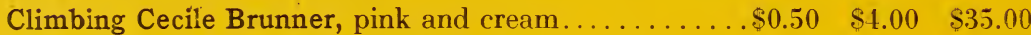

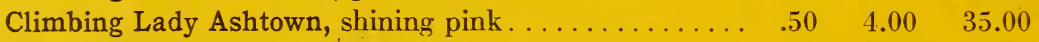

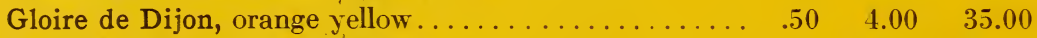

Zephirine Drouhin, bright silvery pink ............. $\quad .50 \quad 4.00 \quad 35.00$ 


\section{HYBRID PERPETUALS AND RUGOSAS}

Mainly budded plants

Alfred Colomb, strawberry red . . . . . . . . . . . . $\ldots \ldots \ldots 35.00$

American Beauty, dark pink, shaded smoky carmine ........ 35.00

Anna de Diesbach, carmine pink

35.00

Baron de Bonstettin, velvety crimson

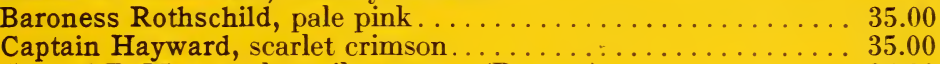

Conrad F. Meyer, clear silvery rose (Rugosa) . . . . . . . . . 35.00

Fisher Holmes, crimson scarlet. . . . . . . . . . . . . . . . 35.00

F. J. Grootendorst, red carnation-like bloom (Rugosa) . . . . . . . 35.00

Frau Karl Druschki, white American Beauty ............. 35.00

General Jacqueminot, crimson - "The Jack Rose" . . . . . . . . 35.00

Georg Arends, delicate pink.

George Dickson, dark red . . . . . . . . . . . . . . . . 35.00

Heinrich Munch, soft pink

J. B. Clark, scarlet crimson

Magna Charta, bright rose pink

Mrs. John Laing, soft pink

Paul Neyron, clear pink.

Prince Camille de Rohan, very dark red . . . . . . . . . . . . 35.00

Ulrich Brunner, cherry red.

No. 2

$\$ 20.00$

20.00

20.00

20.00

20.00

20.00

20.00

20.00

20.00

20.00

20.00

20.00

20.00

20.00

20.00

20.00

20.00

\section{BABY RAMBLERS}

\section{Budded and own root stock}

Cecile Brunner, dainty pink

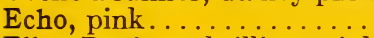

Ellen Poulsen, brilliant pink

Erna Teschendorff, dark red

George Elger, golden yellow

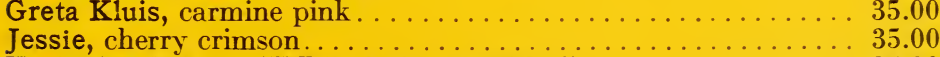

Katharina Zeimet, "White Baby Rambler"............ . 35.00

Maman Levavasseur, "Pink Baby Rambler"............ . 35.00

Miss Edith Cavell, red .......................... 35.00

Mme. Norbert Levavasseur, "Red Baby Rambler"...........

Orleans, brilliant geranium red.

35.00

Triomphe Orleanais, deep crimson. ...................... 35.00

$\$ 18.00$

20.00

20.00

20.00

20.00

20.00

20.00

15.00

20.00

18.00

18.00

18.00

20.00

\section{EXPERT INFORMATION "HOW TO GROW ROSES"}

Up-to-date edition by Robert Pyle is chock-full of practical answers to problems of Planting, Pruning, Protecting, etc. Leading Nurserymen sell this book to their customers. J. Horace McFarland says "more copies bought yearly than total all other Rose books combined." Retails $\$ 2$ per copy, post paid. Trade price $\$ 1.50$, f. o. b. West Grove. Send for sample copy for 10 days' examination. 


\section{THE CONARD-PYLE CO. WEST GROVE, PA. ROBERT PYLE, President}

\section{EVERBLOOMING ROSES (Hybrid Teas and Teas)}

Almost all are Eastern-grown, budded on Multiflora. Prices are per 100. Add 5c. per plant for 10 or more; and 15c. per plant for less than 10, as Roses are put up in bundles of 10 each.

No. 1

Per 100

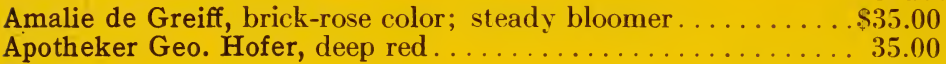

Betty, coppery rose.

35.00

Charles $\mathrm{K}$. Douglas, striking crimson-scarlet.

Cheerful, bright pink

Columbia, lively bright pink; sweet-scented

Constance, rich chrome yellow.

Crimson Queen, rich crimson; very fragrant

Crusader, velvety crimson.

Dorothy Page-Reberts, coppery pink

Duchess of Wellington, saffron yellow; vigorous grower

Ecarlate, scarlet rose.

Edel, ivory white; notable and new

Etoile de France, intense crimson. .

Eugene E. Marlitt, dark red.

Florence Pemberton, clear light pink

Francis Scott Key, light crimson; quite hardy

General McArthur, crimson scarlet.

General Sup. A. Janssen, deep carmine

George C. Waud, distinct vermilion

Golden Emblem, intense yellow; glossy foliage.

Gorgeous, light yellow and orange.

Grace Molyneux, light flesh color

Grange Colombe, soft ivory white.

Gruss an Teplitz, brilliant crimson; vigorous .

Hoosier Beauty, glowing crimson

Humbsldt, light pink.

H. V. Machin, dark red

Janet, salmon flesh.

Jonkeer J. L. Mock, imperial pink

Kaiserin Auguste Viktoria, soft white

Killarney, sparkling pink

Killarney Brilliant, deep pink

Killarney Queen, clear pink

Killarney White, pure white

Konigin Carola, bright rose

Lady Alice Stanley, coral rose

Lady Ashtown, pale rose

Lady Mary Ward, wonderful shade of orange

La France, peach blossom pink.

35.00

Lady Hillingdon, golden yellow

Lieutenant Chaure, velvety crimson

Los Angeles, flame pink.

Louise C. Breslau, shrimp pink, shaded orange . . . . . . . 40.00

Mme. Bardou Job, chrome yellow . . . . . . . . . . . . 40.00

Mme. Butterfly, salmon pink.

Mme. Caroline Testout, clear rich pink.
35.00

35.00

35.00

35.00

35.00

35.00

40.00

35.00

35.00

35.00

35.00

35.00

35.00

35.00

35.00

35.00

No. 2

Per 100

$\$ 25.00$

20.00

20.00

20.00

25.00

20.00

20.00

20.00

25.00

20.00

20.00

20.00

20.00

20.00

20.00

20.00

25.00

25.00

20.00

20.00

20.00

20.00

20.00

20.00

20.00

20.00

20.00

20.00

20.00

20.00

20.00

40.00

40.00

20.00

25.00

20.00

25.00

25.00

25.00

25.00

20.00 


\section{WHOLESALE PRICE LIST \\ SPRING 1926}

\section{EVERBLOOMING ROSES (Continued)}

\begin{tabular}{|c|c|c|}
\hline & $\begin{array}{c}\text { Per } 100 \\
\text { No. } 1\end{array}$ & $\begin{array}{l}\text { Per } 100 \\
\text { No. } 2\end{array}$ \\
\hline Mme. Edouard Herriot, coral red. & & $\$ 25.00$ \\
\hline Mme. Gustave Metz, light pink. . & & 20.00 \\
\hline Mme. Jules Bouche, superb white. &.$\$ 35.00$ & 20.00 \\
\hline Mme. Jules Grolez, satiny pink. . & & 20.00 \\
\hline Mme. Leon Pain, salmon pink. . & 35.00 & 20.00 \\
\hline Mme. Ravary, golden yellow...... & 40.00 & 25.00 \\
\hline Mme. Segond Webər, salmon pink. & 35.00 & 20.00 \\
\hline Marquis de Ganay, silvery pink... & 35.00 & \\
\hline Miss Amelia Gude, creamy yellow . . . . & 40.00 & 25.00 \\
\hline Miss Cynthia Forde, brilliant rose pink. & 35.00 & 20.00 \\
\hline Miss Lolita Armour, chrome yellow. . . . & 40.00 & 25.00 \\
\hline Mrs. Aaron Ward, rich golden fawn . . . . . . . . & & 25.00 \\
\hline Mrs. Ambrose Ricardo, flesh pink and honey yellow. & 40.00 & 25.00 \\
\hline Mrs. Calvin Coolidge, clear buff yellow . . . . . . . . & 40.00 & 25.00 \\
\hline Mrs. Charles E. Russell, rose carmine. & 35.00 & 20.00 \\
\hline Mrs. David M'Kee, creamy yellow....... & & 25.00 \\
\hline Mrs. George Shawyer, clear brilliant rose. & 35.00 & 20.00 \\
\hline Mrs. S. K. Rindge, pure yellow .......... & & 25.00 \\
\hline Mrs. H. R. Darlington, cream-white ............ & 35.00 & \\
\hline Mrs. W. C. Miller, pearly flesh. . . . . . . . . . . . & & 20.00 \\
\hline Pharisaer, cream $\ldots \ldots \ldots \ldots \ldots \ldots \ldots \ldots$ & 35.00 & \\
\hline Prince de Bulgarie, flesh, tinted salmon and saffron. & & \\
\hline Queen of Fragrance, shell pink............... & & 20.00 \\
\hline Queen Mary, bright canary yellow & 40.00 & \\
\hline Red Radiance, cerise red........... & . 35.00 & 20.00 \\
\hline Rose Marie, clear rose pink . . . . . . . & & 20.00 \\
\hline Senateur Mascuraud, clear yellow . . . . . . . . & 40.00 & 25.00 \\
\hline Souv. de Gustave Prat, clear amber yellow. & 40.00 & 25.00 \\
\hline Souv. de Claudius Pernet, sunflower yellow . . . . . . . & & 35.00 \\
\hline Souv. du President Carnot, rosy flesh; shaded white. & 35.00 & 20.00 \\
\hline Una Wallace, bright pink $\ldots \ldots \ldots \ldots \ldots \ldots \ldots$ & . 35.00 & \\
\hline Walter Speed, lemon yellow..... & 40.00 & 25.00 \\
\hline Wm. F. Dreer, silvery shell pink........... & & 25.00 \\
\hline William R. Smith, deep cream, shaded pink. & & 20.00 \\
\hline Willowmere, shrimp pink. ........... & & 20.00 \\
\hline Winnie Davis, attractive apricot pink. . & 35.00 & 20.00 \\
\hline
\end{tabular}

\section{ROSES FOR LINING OUT}

From 21/4-in. pots, except as noted

American Pillar............. \$7.00

Baby Doll, 3-in. pot.......... 10.00

Birdie Blye ............. 7.00

Climbing American Beauty . . . . 7.00

Coronation . . . . . . . . . . 7.00

Dr. Van Fleet................. 7.00

Echo, 3 -in. pot................. 10.00

Erna Teschendorff, 3 -in. pot. . . 10.00

Gardenia .
George Elger, 3-in. pot....... \$10.00

Hugonis ................ . . 35.00

Mary Wallace.............. 25.00

May Queen................ 7.00

Max Graf.................... 25.00

Paul's Scarlet Climbər. . . . . . . . 7.00

Prof. Sargent. . . . . . . . . . . . . . 7.00

Tausendschon............... 7.00

Violet Blue................. 7.00 


\section{OAK BRAND SHRUBS}

Come see our Nursery, and we will stow you a giant Oak to match that in any Nursery anywhere. Here, too, we shall be pleased to stow you OAK BRAND SHRUBS-Sturdy, well-rooted stock carefully graded and better than the ordinary.

Buddleia Variabilis Magnifica.

$2-21 / 2 \mathrm{ft}$.

Bur

Callicarpa Purpurea.

$3-4 \mathrm{ft}$.

Callicarpa Purpurea

$\begin{array}{lll}18 & -24 & \text { in. }\end{array}$

$21 / 2-3 \mathrm{ft}$.

Calycanthus Floridus, 1 yr.

$6-9$

Cornus Sanguinea.

$21 / 2-3$

Deutzia Crenata.

$2-21 / 2 \mathrm{ft}$

Deutzia Crenata

$3-4 \mathrm{ft}$.

Deutzia Gracilis

$\begin{array}{ll}9 & -12\end{array}$

Deutzia Gracilis

Deutzia Gracilis Rosea

$\begin{array}{ll}12 & -18\end{array}$

Deutzia Gracilis Rosea

Deutzia Lemoinei

$\begin{array}{ll}9 & -12\end{array}$

$18-24$ in.

Deutzia Lemoinei

$18 \quad-24$ in.

$2-21 / 2 \mathrm{ft}$.

$2-21 / 2 \mathrm{ft}$.

Deutzia Magnifica

Deutzia Magnifica

\section{New. By far best} of all Deutzias.

$21 / 2-3 \mathrm{ft}$.

$3-4 \mathrm{ft}$.

Deutzia Pride of Rochester

$2-21 / 2 \mathrm{ft}$.

Deutzia Pride of Rochester

$21 / 2-3 \mathrm{ft}$.

Euonymous Japonica.

12 in.

Forsythia Spectabilis, 2 yr.

Forsythia Spectabilis New. Has proved
most desirable.

Hibiscus, Althea Banner.

Hibiscus, Althea Banner.

$\ldots \ldots \ldots \ldots 12 \quad-18$

Hibiscus, Althea Double

.......... 18

(1)......1 1

Hibiscus, Althea Jeanne D'Arc 2 yr.....

Hibiscus, Althea Jeanne D'Arc Hibiscus, Althea Wm. R. Smith Magnificent
Hibiscus, Althea Wm.R. Smith new variety.

Hibiscus, Althea Wm. R. Smith

Honeysuckle Morrowi . . ...........

Honeysuckle Morrowi.

Honeysuckle Morrowi.

Honeysuckle Tatarica White ..........12

Honeysuckle Tatarica White..........

Hydrangea P. G

Hydrangea P. G.................... 18

Hydrangea P. G., 3 yr. . . . . . . . . . .

Hydrangea Arborescens Grand., extra

bushy................... 21/2-3

Philadelphus Avalanche ........... 12 -18

Philadelphus Avalanche ........... 21/2- 3

Philadelphus Avalanche ............ 3

Philadelphus Bouquet Blanc, 1 yr ..... 9

$\stackrel{1}{80.30}$

10

.45

.30

.40

.25

.30

.25

.30

.25

.35

.25

.50

.45

.50

.60

.50

.60

.25

.30

.25

.60

.85

.20

.30

.25

.30

.25

.30

.45

.60

.25

.30

.35

.25

.30

.25

.35

.45

2.50

4.00

2.50

3.50

2.00

2.50

2.00

2.50

2.00

3.00

2.00

4.50

4.00

4.50

5.50

4.50

5.50

2.00

2.50

2.25

5.50

8.00

1.75

2.50

2.00

2.50

2.00

2.50

4.00

5.50

2.00

2.50

3.00

2.00

2.50

2.00

3.00

4.00

100

$\$ 20.00$

35.00

20.00

30.00

15.00

20.00

16.00

20.00

17.50

25.00

17.50

40.00

35.00 .

40.00

50.00

40.00

50.00

16.00

20.00

20.00

50.00

75.00

15.00

20.00

15.00

20.00

15.00

20.00

35.00

50.00

18.00

20.00

25.00

15.00

22.00

15.00

25.00

35.00

4.00

35.00

2.00

2.50

15.00

in. $\quad .25$

ft. $\quad .35$

3.00

20.00

2.00
1000

$\$ 180.00$

125.00

150.00

200.00

150.00

375.00 


\section{OAK BRAND SHRUBS-Continued}

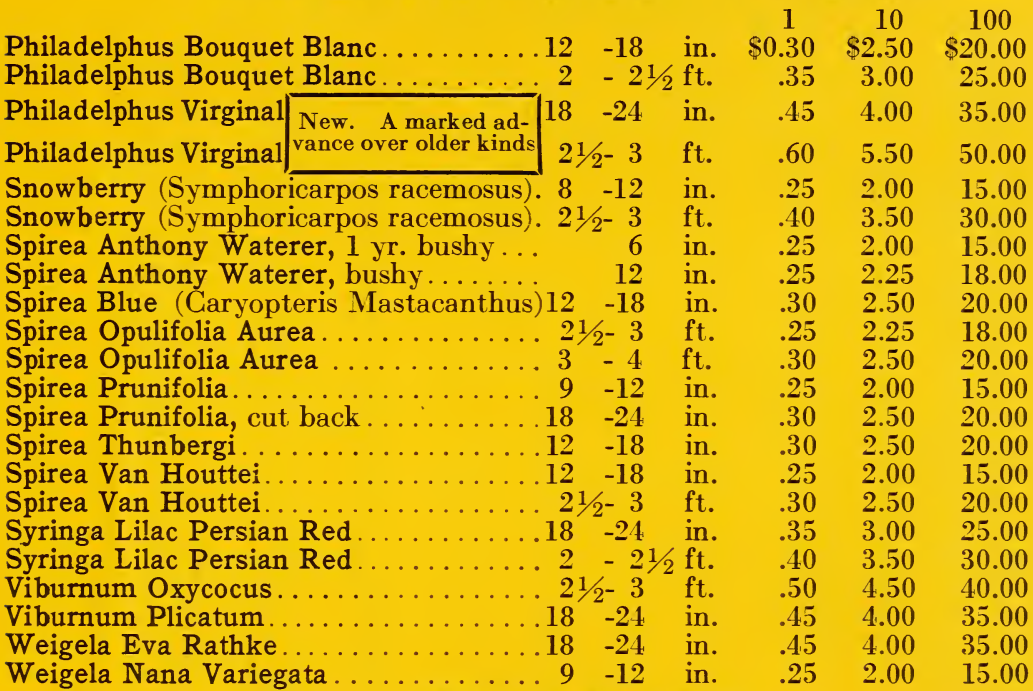

HEDGE PLANTS

\section{BOX BARBERRY (Berberis Thunbergi Minor)}

New dwarf compact form of the popular Berberis Thunbergi. Hardy. May be trimmed any time without harm. Especially useful for edging. Insures a neat unbroken border. Makes a nice hedge 6 to 15 in. high. Very decorative, when trimmed into formal balls or pyramids. Can be planted as a foreground shrub or used in foundation landscape work.

\begin{tabular}{|c|c|c|c|c|c|}
\hline \multirow{4}{*}{ SELLING } & Size & 1 & 10 & 100 & 1000 \\
\hline & 1 yr., $6-8$ in. & $\$ 0.20$ & $\$ 1.50$ & $\$ 10.00$ & $\$ 90.00$ \\
\hline & 2 yr., $8-12$ in. & .22 & 1.80 & 12.50 & 100.00 \\
\hline & $2 \mathrm{yr} ., 12-15$ in. & .25 & 2.00 & 15.00 & 120.00 \\
\hline & 3 yr., $12-15$ in. & .25 & 2.25 & 18.00 & 160.00 \\
\hline & $4 \mathrm{yr} ., 18-24$ in. & .45 & 4.00 & 35.00 & \\
\hline
\end{tabular}

Write for prices in larger quantities. Size

Barberry Thunbergi, 1 yr........... 9 -12

Barberry Thunbergi, 2 yr........... 12 -18

Barberry Thunbergi.............. 18 -24

Berberis Wilsonae, 1 yr., bushy....... 6

Berberis Wilsonae, exceptionally good. .12

Box Edging (Buxus Semp. Suff.), 21/4 in. pots

Box Edging (Buxus Semp. Suff.), 3 in. pots

California Privet (L. Ovalifolium), 1 yr., 12-18 in.

California Privet (L. Ovalifolium),..... 18-24 in.

California Privet (L. Ovalifolium) ...... 21/2- 3

Ibolium Privet (L. Ibolium) . . . . . . . . 21/2- 3

Lodense Privet (L. Nanum Compactum).12 -18

\begin{tabular}{lrrrr} 
& \multicolumn{1}{c}{1} & \multicolumn{1}{c}{10} & \multicolumn{1}{c}{100} & \multicolumn{1}{c}{1000} \\
in. & $\$ 0.15$ & $\$ 1.20$ & $\$ \$ 7.50$ & $\$ 60.00$ \\
in. & .20 & 1.50 & 10.00 & 75.00 \\
in. & .25 & 2.25 & 15.00 & 120.00 \\
in. & .35 & 3.00 & 25.00 & \\
in. & .45 & 4.00 & 35.00 & \\
& & 1.20 & 7.00 & 60.00 \\
& & 1.50 & 10.00 & 80.00 \\
& .10 & .50 & 3.50 & 30.00 \\
& .10 & .75 & 5.00 & 37.50 \\
ft. & .12 & .80 & 6.00 & 50.00 \\
ft. & .20 & 1.50 & 12.00 & 100.00 \\
in. & .20 & 1.50 & 12.00 & 100.00
\end{tabular}

Useful for low hedge or border.

Stands shearing well. 


\section{FIELD VINES}

Akebia Quinata, 2 yr

\begin{tabular}{|c|c|c|c|c|}
\hline & & 1 & 10 & 100 \\
\hline & & $\$ 0.25$ & $\$ 2.00$ & $\$ 15.00$ \\
\hline-3 & $\mathrm{ft}$. & .35 & 3.00 & 25.00 \\
\hline $1 / 2-3$ & $\mathrm{ft}$. & .25 & 2.25 & 18.00 \\
\hline-4 & ft. & .30 & & \\
\hline$-21 / 2$ & $\mathrm{ft}$. & .25 & 2.00 & 15.00 \\
\hline-4 & $\mathrm{ft}$. & .35 & 3.00 & \\
\hline-18 & in & .2 & 2.0 & \\
\hline$-21 / 2$ & $\mathrm{ft}$. & .2 & 2.25 & 18.00 \\
\hline & in. & .2 & 2.00 & \\
\hline-12 & in. & .30 & 2.50 & 0.00 \\
\hline & & .35 & 3.00 & 5.00 \\
\hline & & .25 & 2.25 & 18.00 \\
\hline & & & 2.50 & \\
\hline 6- & ft. & & 2.00 & 15.00 \\
\hline & $\mathrm{ft}$. & & 2.25 & .00 \\
\hline & & & 2.2 .5 & \\
\hline $1 / 2-3$ & ft. & .30 & 2.50 & 20.00 \\
\hline & & & 2.75 & \\
\hline & & & 3.00 & 27.00 \\
\hline & & .27 & 2.25 & 18.00 \\
\hline $21 / 2$ & $\mathrm{ft}$ & .30 & 2.50 & 20.00 \\
\hline-2 & $\mathrm{ft}$ & .35 & 3.00 & 25.00 \\
\hline
\end{tabular}

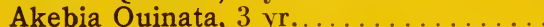

Ampelopsis Quinquefolia, 2 yr.........

Ampelopsis Quinquefolia, 3 yr. .......

Celastrus Scandens, 2 yr...........

Celastrus Scandens, 3 yr............

Chinese Matrimony Vine, 2 yr........

Chinese Matrimony Vine, 3 yr........

Euonymous Radicans, 2 yr...........

Euonymous Radicans, 3 yr., bushy. . . . .

Euonymous Radicans Variegata, 3 yr., bushy

Honeysuckle Evergreen, 2 yr.

Honeysuckle Evergreen, 3 yr. . .

Honeysuckle Golden, 2 yr.

Honeysuckle Golden, 3 yr.

Honeysuckle Halliana, 2 yr.

Honeysuckle Halliana, 3 yr.

Honeysuckle Red Coral, 2 yr.

Honeysuckle Red Coral, 3 yr.

Wistaria Magnifica, 1 yr............ 12 -18 in.

Wistaria Magnifica............. $2-21 / 2 \mathrm{ft}$.

Wistaria Magnifica

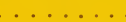




\section{WHOLESALE PRICE LIST \\ SPRING 1926}

\section{LINING OUT STOCK-SHRUBS}

\begin{tabular}{|c|c|c|c|c|}
\hline & & 10 & 100 & 1000 \\
\hline belia Grandiflora, extra fine field grown ... . . & 3 -in. pot & $\$ 2.50$ & $\$ 20.00$ & $\$ 180.00$ \\
\hline lia Grandiflora, well-established plants. . . . . & $31 / 2$-in. pot & 3.00 & 25.00 & 225.00 \\
\hline elia Grandiflora, extra fine field grown...... & 4 -in. pot & 3.50 & 30.00 & 275.00 \\
\hline dleia Asiatica . . . . . . . . . . . . . & $21 / 4$-in. pot & 1.00 & 7.50 & 60.00 \\
\hline uddleia Magnifica . . . . . . . . . . . . . . . & $21 / 4$-in. pot & 1.00 & 7.00 & 60.00 \\
\hline otoneaster Horizontalis, nice long branches. . . & $3^{-i n}$. pot & 3.00 & 25.00 & \\
\hline eutzia Gracilis, pot-grown . . . . . . . . . . . & & & 6.00 & \\
\hline 1tzia Gracilis Rosea, pot grown. . . . . . . . . & & & 6.00 & \\
\hline eutzia Lemoinei, pot-grown. . . . . . . . . . . . & & & 6.00 & \\
\hline uonymous Japonica.......... . & $21 / 4$-in. pot & 1.00 & 7.00 & 60.00 \\
\hline Forsythia Spectabilis, pot-grown. & & & 12.00 & \\
\hline Hibiscus Althea Jeanne D'Arc . . . . . . . . . . . . & 21/4-in. pot & 1.50 & 10.00 & \\
\hline Hibiscus Althea Wm. R. Smith, magnificent: new & $2 \frac{1}{4}$-in. pot & 4.00 & 35.00 & \\
\hline 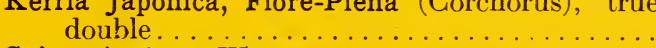 & $21 / 4$-in. pot & 1.00 & 7.00 & 60.00 \\
\hline 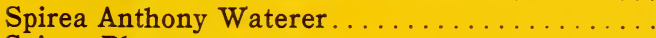 & $21 / 4$-in. pot & 1.00 & 7.00 & 60.00 \\
\hline 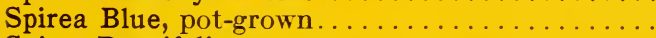 & & 1.00 & 6.00 & \\
\hline ea Prunifolia............. & $21 / 4$-in. pot & 1.00 & 7.00 & 60.00 \\
\hline ea Van Houttei & $21 / 4$-in. pot & 1.00 & 7.00 & 60.00 \\
\hline
\end{tabular}

\section{Viburnum Plicatum for Growing On}

For years one of our specialties. Plants now for sale are exceptionally good and totally different from any offered elsewhere. Extra strong and well-rocted. 3 -in. pots $\$ 17.50$ per $100 ; \$ 150$ per 1000

\section{LINING OUT STOCK-VINES}

\begin{tabular}{|c|c|c|c|c|}
\hline & & 10 & 100 & 1000 \\
\hline Ampelopsis Veitchii. . . . . . . . . . . . . . & $21 / 4$-in. pot & $\$ 1.50$ & $\$ 9.00$ & $\$ 75.00$ \\
\hline Ampelopsis Veitchii, well furnished and bushy. & 4 -in. pot & 3.00 & 25.00 & \\
\hline 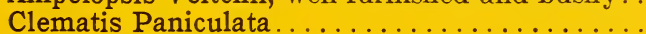 & $21 / 4$-in. pot & 1.50 & 9.00 & 75.00 \\
\hline Euonymous Radicans........ & $21 / 4$-in. pot & 1.20 & 7.00 & 55.00 \\
\hline Euonymous Radicans Variegata. & $21 / 4$-in. pot & 1.20 & 7.00 & 55.00 \\
\hline Euonymous Vegetus.......... & $21 / 4$-in. pot & 1.20 & 8.00 & 75.00 \\
\hline English Ivy (Hedera Helix) & $21 / 4$-in. pot & 1.00 & 6.00 & 50.00 \\
\hline
\end{tabular}

\section{LINING OUT STOCK-EVERGREENS}

Note.-These young Evergreens have an exceptionally good reputation among Nurserymen and Florists. Being pot-grown, they are especially well-rooted, and we believe will surpass any offered elsewhere.

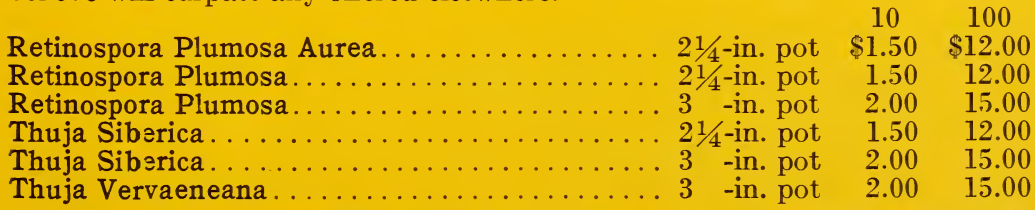

Note.-We have excellent facilities for propagating young Evergreens. If interested in having us grow special lots, please write us. 


\section{צ CANNAS-AMERICA'S BES'T}

Green foliage except as noted

The Cannas from which the $(\mathbf{5})$ is omitted are the only varieties we offer that were not originated by the late Antoine Wintzer and introduced by us.

\section{New Beauty Dominates Canna World}

For more than 30 years buyers of Cannas have turned confidently to West Grove, finding in the work of the late Antoine Wintzer, the best varieties in commerce. Good Cannas, one by one, appeared from the skillful hands of this master-producer. But still his clients waited eagerly, unsatisfied, expecting the one superior variety. Suddenly, with a burst of splendid luck, he introduced the best pink Canna of his whole 30 years' hybridizing-5 Mrs. Pierre S. duPont. In the tremendous wave of approval that followed, Canna-men recognized at last the long-looked for, much-anticipated variety.

Where, except in this wonderful Canna, will you find such pure rich color-a vivid watermelon pink? A large flower head with ruffled petals. Firm, erect stem, gigantic foliage. A sturdy grower standing up well under hot sun. Truly, leaders in the Canna World are delighted with this attractive-growing eyecatching variety.

The roots we offer you are extra heavy, plump 2 to 3 eye divisions. Prices are at a new low level. Follow the judgment of keen business men-make up your order for Mrs. duPont now.
Each
$\$ 1.15$
10
$\$ 11.00$
100
$\$ 100.00$

As in pinks, so in other colors, especially yellows, do the following Cannas represent the cream of America's best.

5 American Beauty, $5 \mathrm{ft}$. Richest colored Canna we know, oriental carmine. Large flowers.

$10 \quad 100 \quad 1000$

5 Apricot, $4 \mathrm{ft}$. Flower has marvelous durability. Unlike most

Cannas this rich apricot variety is suitable for cutting . . . $1.00 \quad 7.00$
Baltimore, $3 \mathrm{ft}$. Rose-pink. Exceptionally large compact

$\$ 90.00$ flowers, borne well above foliage. All-season bloomer. (New) 2.50

Y5 Beacon, $4 \mathrm{ft}$. Medium sized flowers of rich cardinal red.....

Buttercup Imp., $3 \mathrm{ft}$. Marvelous improvement on Buttercup

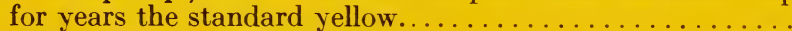

$5.00 \quad 40.00$

Y Candalabra, $5 \mathrm{ft}$. Three to five branches on each stalk. Flowers torch-like flames of orange scarlet......... 1.50

5 City of Portland, $3 \frac{1}{2} \mathrm{ft}$. Magnificent pink bedder... . . . . . 1.50 Cupid, $3 \mathrm{ft}$. Fascinating lilac rose. Bountiful bloomer. (New). 4.00 Edward W. Bok, $3 \mathrm{ft}$. A great favorite. Immense flower head. Color soft light rose, petals edged cream. (New) . . . . . 4.00

Prices are for plump dormant divisions, 2 to 3 eyes, plus roots. Prices of plants from 3-in. pots, ready May 1st, are same as for dormant roots except $\$ 3$ per 100 must be added on all varieties priced below at less than $\$ 8$ per hundred. 


\section{si CANNAS-Continued}

Y Gaiety, $5 \mathrm{ft}$. Well-named. Variously colored flowers,

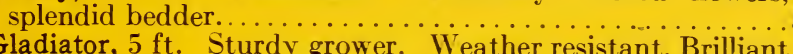
yellow, dotted red..

צ Gladioflora, $31 / 2 \mathrm{ft}$. Crimson, changing to rose. Exceptionally

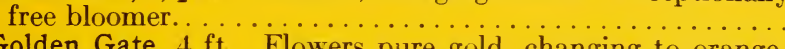

5 Golden Gate, $4 \mathrm{ft}$. Flowers pure gold, changing to orange-

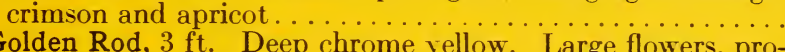

45 Golden Rod, $3 \mathrm{ft}$. Deep chrome yellow. Large flowers, pro-

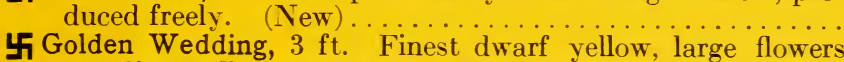
standing well above foliage. Widely used by Park men for bedding.

Hungaria, $31 / 2 \mathrm{ft}$. Sparkling rose pink. Old favorite.

King Humbərt, $4 \mathrm{ft}$. Most popular bronze-leaved variety. Orange scarlet, streaked crimson.

צ $\mathrm{f}$ King Midas, $5 \mathrm{ft}$. A true Canna aristocrat. Very large flower of glittering gamboge-yellow. Outstanding variety

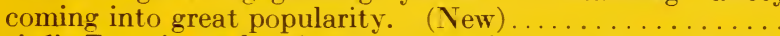

乌 Laird's Favorite, $4 \mathrm{ft}$. A most attractive new orange-yellow.

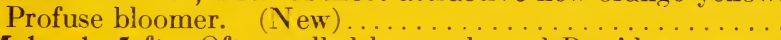

$\mathbf{5}$ Mohawk, $5 \mathrm{ft}$. Often called bronze-leaved President, poppy

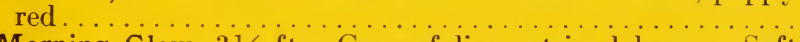
Morning Glow, $3 \frac{1}{2} \mathrm{ft}$. Green foliage striped bronze. Soft shell pink.

Morning Star, $5 \mathrm{ft}$. Color is madder carmine; petals edged yellow.

צ. $\mathrm{M}$

Mrs. Alfred F. Conard, $4 \mathrm{ft}$. On the market for 15 years, yet demand always exceeds supply. Firm, lasting, salmon-pink flowers.

\begin{tabular}{|c|c|c|c|}
\hline Pierre S. du Pont, $4 \mathrm{ft}$. Wintzer's 1 & 10 & 100 & 1000 \\
\hline see details top of page $12 \ldots \$ 1.25$ & $\$ 11.00$ & $\$ 100.00$ & (By request) \\
\hline
\end{tabular}

Y Mrs. Antoine Wintzer, $4 \mathrm{ft}$. Pure, warm, unshaded yellow.

$10 \quad 100 \quad 1000$

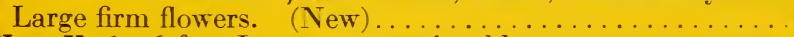

近 New York, $6 \mathrm{ft}$. Immense carmine blooms on erect stems. Bronze foliage .............................................
Nokomis, $5 \mathrm{ft}$. Large vivid crimson flowers.

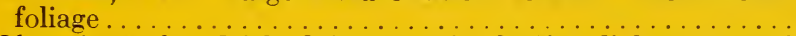

乌) Olympic, $5 \mathrm{ft}$. Rich Oriental red, shading lighter toward center. Free bloomer.

The Cannas from which the (4) is omitted are the only varieties we offer that were not originated by the late Antoine Wintzer and introduced by us. 


\section{THE CONARD-PYLE CO. WEST GROVE, PA. \\ ROBERT PYLE, President}

\section{ч}

Y $\mathrm{Palm}$ Beach, $3 \frac{1}{2} \mathrm{ft}$. Broad, long-petaled flowers of maize yellow, with faint bush of pink. Really a rival of finest orchids. Eye-catching and destined to great popularity. (New) ................................................

\section{$\$ 3.50$}

5.00

$\$ 250.00$

4 Panama, $3 \mathrm{ft}$. Large flowers of rich orange red. Good bedder
대 Paulina, $3 \mathrm{ft}$. Large firm, lasting flowers of lemon yellow. (New) . . . . . . . . . . . . . . . . . . . . . . . .

$3.50 \quad 30.00$

$1.20 \quad 10.00$

Queen Helen (Yellow King Humbert), $5 \mathrm{ft}$. Flowers yellow, dotted red. Foliage green except sometimes one-half is

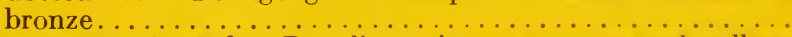

If Razzle Dazzle, $4 \mathrm{ft}$. Dazzling crimson-maroon and yellow. Very pretty. (New) . . . . . . . . . . . . . . .

5 Radio, $4 \mathrm{ft}$. Primrose white. Cannas this color very scarce. (New).

乐 $\mathrm{r}$ Rosea Gi

Statue of Liberty, $6 \mathrm{ft}$. Immense leaves of rich, bronzy

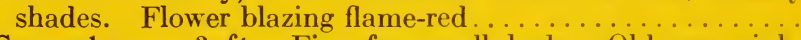

צi Susquehanna, $3 \mathrm{ft}$. Fine for small beds. Old-rose pink.

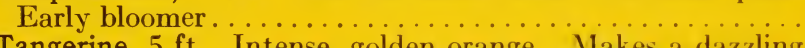

צi Tangerine, $5 \mathrm{ft}$. Intense, golden orange. Makes a dazzling

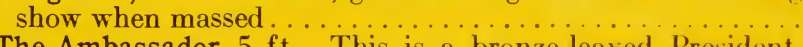

The Ambassador, $5 \mathrm{ft}$. This is a bronze-leaved President. Foliage pretty bronze. Blooms red

60.00

$1.00 \quad 35.00 \quad 300.00$

$3.00 \quad 25.00 \quad 225.00$

$2.00 \quad 15.00$

$1.50 \quad 10.00$

90.00

$1.50 \quad 12.00 \quad 100.00$

$3.00 \quad 2.5 .00 \quad 225.00$

$1.00 \quad 25.00 \quad 200.00$

Y5 The President, $4 \mathrm{ft}$. Far and away superior to any other red Canna produced. Used by practically every Park

Board in the country. Makes wonderful beds of glowing

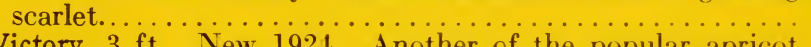

Yi Victory, $3 \mathrm{ft}$. New 1924. Another of the popular apricot shades. Flowers medium size. (New)

50.00

S5 Vulcan, $3 \mathrm{ft}$. Medium-sized flowers, cardinal-red in color. Splendid when massed. (New)

$2.00 \quad 15.00 \quad 125.00$

$\begin{array}{lll}2.50 & 20.00 & 180.00\end{array}$

צi Wabash, $4 \mathrm{ft}$. Rich carmine pink, great broad-petaled flowers

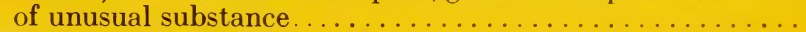

Y W Wintzer's Colossal, $5 \mathrm{ft}$. Average flowers more than cover a man's hat. Color a striking vivid scarlet............ Wyoming, $7 \mathrm{ft}$. Foliage rich purplish bronze. Very large

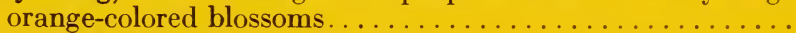
Cannas in Assortment. Good colors and nice stock. $2.50 \quad 20.00$ $\begin{array}{lll}1.00 & 6.00 \quad 50.00\end{array}$ $\begin{array}{lll}.5 & 4.00 \quad 35.00\end{array}$ $\begin{array}{lll}.50 & 4.00 \quad 35.00\end{array}$ The Cannas from which the (4) is omitted are the only varieties we offer that were not originated by the late Antoine Wintzer and introduced by us.

\section{HOW TO PLANT CANNAS IN CIRCULAR BEDS}

Plant 1 in the center, 6 in the first circle, 12 in the second circle then 18,24 , etc., according to the size of the bed with the circles, and the plants in the circles 18 inches apart. A bed 4 feet in diameter takes 7 Cannas; a 7 -foot bed, 19 cannas; a 10 -foot bed, 37 Cannas; a 16-foot bed, 91 Cannas. 
FROM
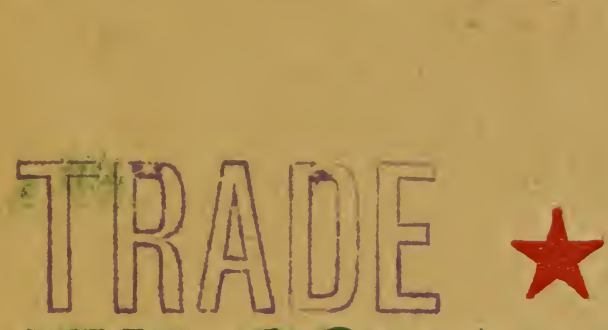

THE CONARD-PYLE

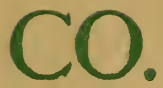

Star Rose Growers

Robert Pyle, Pres.

West Grove, $\mathrm{Pa}$. 


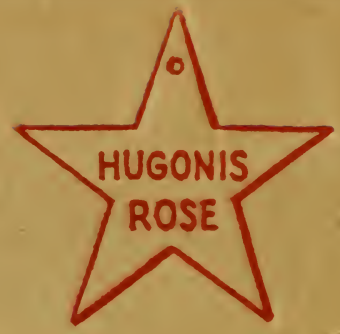

ON EVERY STAR ROSE

IS A CELLULOID TAG

OUR GUARANTEE THAT STAR ROSES

WILL BLOOM OR YOUR MONEY BACK 


\section{CHRYSANTHEMUMS}

Good Pot-plants, $\$ 12.00$ per $100 ; \$ 100.00$ per 1000

Brown Bessie (Pompon), Gold and brown Cherry Red (Pompon), Crimson maroon Little Bob (Pompon), Small bronze flowers Myers Perfection (Button), Snowy-white
Oconto (Large Flowering), Early white Unaka (Incurved), The long-sought early October pink

Uvalda (Pompon), Glistening white Assorted Chrysanthemums. Good kinds

\section{OTHER PERENNIALS}

Pachysandra, ground cover .

Pachysandra, ground cover................ Phlox Champs Elysee, magenta............ yr. fld. Phlox Mrs. Jenkins, white................ yr. fld. Platycodon (Balloon flower) Blue............ Yucca Filamentosa (Adams Needle) .......... yr. fld. Yucca Filamentosa (Adams Needle) ........... 3 yr. fld.

\begin{tabular}{rrr}
10 & \multicolumn{1}{|c}{100} & 1000 \\
$\$ 1.00$ & $\$ 7.00$ & $\$ 60.00$ \\
1.00 & 7.00 & \\
1.00 & 7.00 & \\
1.00 & 7.00 & 60.00 \\
1.50 & 10.00 & \\
3.00 & 25.00 &
\end{tabular}

\section{DAHLIAS}

Field Clumps divided; generous divisions

These are an over-supply of varieties which were thoughtfully selected by Dahlia Specialists from a list of 700 kinds.

American Beauty (Show), gorgeous wine crimson $\ldots . . . . . .200$

Augus Megar (Show), large fine lavender . . . . . . . . . . . 2.00

Break O'Day (Dec.), clear sulphur yellow.............. 3.00

Eckford Century (Sgl.), immense pure white................ 1.25

Ellen Kelley (Peony), long-stemmed golden yellow .......... 2.50

Jack Rose (Dec.), deep crimson ..................... 1.00

Le Grand Manitou (Dec.), gigantic white, striped violet . . . . . . 2.00

Libelle (Cactus), clear deep purple................... 1.00

Lilly Grand (Dec.), large white..................... 5.00

Man Friday (Sgl.), deep maroon . . . . . . . . . . . . . . 1.25

Maurice Rivoire (Coll.), rich crimson; white collar............ 1.50

Mina Burgle (Dec.), finest crimson; excellent for cutting. . . . . . 2.00

Mrs. Ella Cline (Dec.), all-around pure white.............. 4.00

Mrs. Newell D. Atwood (Peony), gorgeous deep crimson-red . . . 2. 2.50

Mons. Le Normand (Dec.), rich golden yellow............. 6.00

Painted Beauty (Sgl.), garnet, striped maroon; tipped white.... 1.25

Princess Juliana (Dec.), one of finest whites for cutting ...... 1.50

Red Hussar (Show), rich cardinal red . . . . . . . . . . . . . 1.25

Rose Pink Century(Sgl.), very popular; rose pink ........... 1.00

Sylvia (Dec.), white; heavy pink edge.................... 1.00

Virginia Lee (Coll.), deep velvety carmine; edged sulphur yellow. 2.00

Walker (Dec.), deep lavender pink .................. 1.50

Wilhelm Miller (Dec.), brilliant purple; good ............. 1.00

Per 100

$\$ 12.00$

15.00

20.00

8.00

12.00

15.00

5.00

40.00

6.00

8.00

12.00

35.00

15.00

50.00

6.00

12.00

8.00

6.00

6.00

12.00

8.00

5.00

\section{FRENCH HYDRANGEAS}

4 -in. pots, $\$ 15.00$ per 100

Mme. E. Mouillere, White

Mme. E. Chautard, Early Pink Mousseline, Mauve

Exceptionally well rooted but chiefly single bloom stems. Average 12". Excellent for pinching back and making rousing big bushes for next year. 

